\title{
Ein frühweichselzeitliches Profil aus der Niederterrasse der mittleren Lahn (Weimar-Niederweimar, Hessen) - geologische, pollenanalytische und makrorestanalytische Untersuchungen
}

\author{
Holger Freund \& Ralf URZ *
}

Freund, H. \& URz, R. (2000): Ein frühweichselzeitliches Profil aus der Niederterrasse der mittleren Lahn (Weimar-Niederweimar, Hessen) - geologische, pollenanalytische und makrorestanalytische Untersuchungen. - Eiszeitalter und Gegenwart, 50: 107-123; Hannover 2000.

Keywords: Niederterrasse, Early Weichselian, Interstadial, Brörup, palaeoclimate, Lahn valley, Germany.

Kurzfassung: Die Niederterrasse der mittleren Lahn ist in mindestens drei Aufschotterungsphasen zu gliedern, die dem Weichsel-Frühglazial, dem Weichsel-Pleniglazial und dem ausgehenden Weichsel-Spätglazial (Jüngere Dryas) zuzuordnen sind. Die Paläo-Rinnen im unteren Schotterkörper haben nach den palynologischen und karpologischen Untersuchungen ein brörupzeitliches Alter, wobei aber nicht die gesamte Vegetationsabfolge überliefert ist. Als Bindeglied zwischen dem nördlichen Mitteleuropa und dem Alpenvorland kommt den brörupzeitlichen Profilen aus dem Lahntal von Weimar-Niederweimar eine überregionale Bedeutung bei der Beurteilung des Paläoklimas zu. Pflanzensippen mit subozeanischer Verbreitungstendenz belegen, dass das Klima während des Brörup-Interstadials nicht extrem kontinental getönt war. Die Mittelgebirgszone bildete offenbar die nördliche Verbreitungsgrenze wärmeliebender Laubgehölzc (Eiche, Ulme), wobei die Länge der Vegetationsperiode sowie die Niederschlagsverhältnisse mögliche Ursachen für eine Begrenzung der Ausbreitung nach Norden gewesen sein könnten.

[Early Weichselian deposits from the Niederterrasse of the middle reach of the Lahn river (Weimar-Niederweimar, Hessen) - geological, palynological and macrofossil investigations]

Abstract: Three phases of sediment accumulation are recognised in the Niederterrasse of the middle reach of the river Lahn in Hessen, northern central Germany. These relate to the Weichselian, and individually to the

\footnotetext{
*Anschriften der Verfasser: Dr. H. FREund, Institut für Geobotanik, Universität Hannover, Nienburger Str. 17, D-30167 Hannover; freund@mbox.geobotanik.unihannover.de; DR. R. URz, Institut für Physische Geographie, Professur für Bodenkunde der Universität, Postfach 111932, D-60054 Frankfurt am Main; urz@em.unifrankfurt.de
}

Early- and Pleniglacial periods and the end phase of the Late Glacial (Younger Dryas), respectively. By means of pollen and plant macrofossil analyses carried out on palaeochannel deposits that occur in the lower part of the gravel bed, correlation with the Brörup interstadial was established but a complete Brörup sequence was not represented. This new site is of significance due to its intermediate location between northern central Europe and the Alpine foreland, and so provides fresh evidence of climatic conditions in the Early Weichselian. The presence of species with present-day suboceanic distribution patterns indicates that the climate during the Brörup interstadial was less continental. The uplands of the Mittelgebirge were probably the northern limit of thermophilous trees such as oak and elm. A relatively short growing season as well as reduced precipitation are suggested as the main factors that limited the northerly and easterly extension of the more warmth-demanding tree species.

\section{Einleitung}

Der Kiestagebau im mittleren Lahntal schafft seit vielen Jahren großflächige Aufschlüsse, die den Aufbau der quartären Talfüllung zeigen. Südlich von Marburg sind in der Kiesgrube Weimar-Niederweimar (TK 25 Blatt 5218 Niederwalgern, Abb 1) die fluvialen Sedimente der weichselzeitlichen Niederterrasse bis in eine Tiefe von ca. $12 \mathrm{~m} \mathrm{zu}$ gänglich. An der Basis dieser Ablagerungen treten gelegentlich in flachen Rinnenpositionen Mudden und Niedermoortorfe auf, die teilweise in das Weichsel-Frühglazial (Huckrifde 1982; Urz 1995) beziehungsweise in das Eem-Interglazial (HuckRIEDE 1972; URz 1995) eingeordnet wurden. Kriterien für diese Einstufungen waren lithostratigraphische und makrorestanalytische Befunde; pollenanalytische Bearbeitungen wurden bislang nicht durchgeführt, bzw. sie standen noch aus. Im Zuge der Kiesgrubenerweiterung konnten nahe der Quartärbasis mehrere neue Profile bearbeitet werden. Die zeitliche Einstufung der Sedimente sowie die Rekonstruktion der Vegetations- und Klimaentwicklung stand dabei im Vordergrund der vorliegenden Untersuchung. 

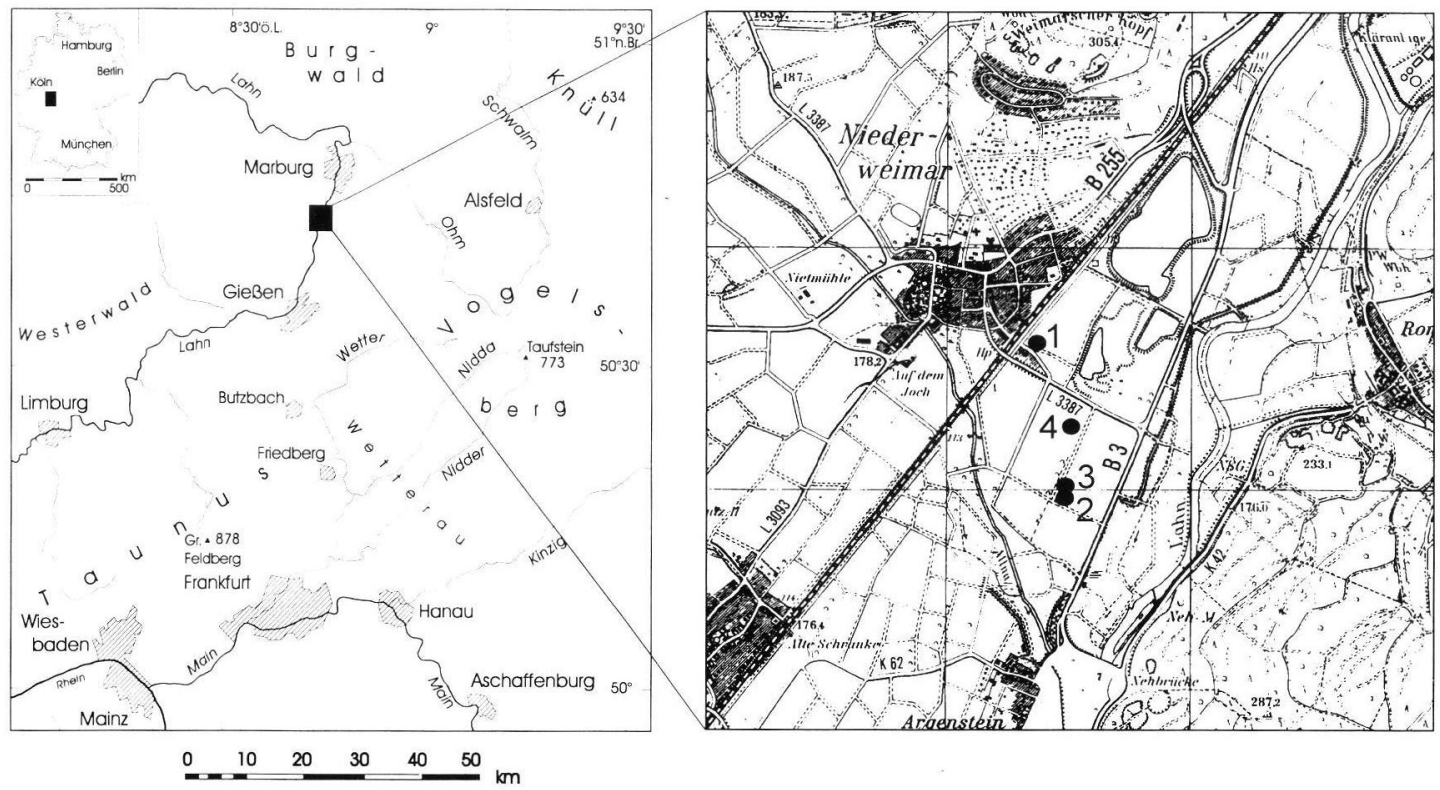

Abb. 1: Kiesgrube Weimar-Niederweimar im mittleren Lahntal mit Lage der Profile I-IV.

Fig. 1: Location of the gravel pit Weimar-Niederweimar in the middle reach of the river Lahn and pollen profiles IIV.

\section{Geologie der Niederterrasse im mittleren Lahntal bei Weimar-Niederweimar}

Die Lahn folgt in ihrem mittleren Abschnitt bei Marburg einer Talzone des hessischen Mittelgebirges, die sich zwischen dem Rheinischen Schiefergebirge im Westen und der Hessischen Senke im Osten erstreckt. Diesem Verlauf liegt eine rheinisch ausgerichtete und im Jungtertiär/Altquartär entstandene Grabenstruktur zugrunde (HöLtiNG \& Stengel-RutKowski 1964, Huckriede 1972). Die Glicderung der pleistozänen Terrassen des Marburger Lahntals erfolgte zuletzt durch HeINE (1970), wobei die Schotter der Talfüllung den jüngsten Terrassenkomplex bilden. Sie wurden bislang, gemeinsam mit der 2-4 m über dem Talbodenniveau gelegenen Terrasse, als weichselzeitliche Bildungen angesprochen und mit der Niederterrasse des Rheins parallelisiert. Für eine erste, detailliertere Gliederung der Niederterrasse konnte URz (1995) die guten Aufschlussverhältnisse durch den Kiesabbau im gesamten mittleren Lahntal nutzen. Lithostratigraphisch lassen sich mehrere Akkumulationsphasen unterscheiden, denen jeweils Erosionsereignisse vorausgingen. Hinzu kommt, dass den Schottern in unterschiedlichen Tiefen Muddehorizonte und Niedermoortorfe zwischengeschaltet sind, die eine bio- und chronostratigraphische Gliederung erlauben (Abb. 2).
Über rotvioletten, mürben Arkosen und Sandsteinen des Perm setzt die Sedimentabfolge der Niederterrasse mit ca. $1 \mathrm{~m}$ mächtigen, groben Sockelschottern ein. Über diesen Kiesen liegen, in unterschiedlicher Höhenlage, mit Mudden und stark kompaktierten Niedermoortorfen verfüllte PaläoRinnen. Auf Grundlage ihrer stratigraphischen Position und nach Auswertung pflanzlicher Makroreste wurden sie, vorbehaltlich weiterer Untersuchungen, zunächst dem Weichsel-Frühglazial (HuCKriede 1982; Urz 1995), stellenweise auch dem Ende des Eem-Interglazials (Kiesgrube Weimar-Niederweimar: Hainbuchen-FichtenZeit, Huckriede 1972; Kiesgrube NiederwalgernRoth: Fichten-Kiefern-Tannen-Zeit, Urz 1995) zugeordnet.

Die hier vorgelegten neuen Profile von Paläo-Rinnen aus der Kiesgrube Weimar-Niederweimar sind in den untersten Abschnitt eines 4-5 m mächtigen, gelblichen Schotterkörpers eingeschaltet. Dieser ist durch Fein- bis Mittelkies und Sand charakterisiert. Im Hangenden folgt über einer markanten Erosionsdiskordanz eine ca. $3 \mathrm{~m}$ mächtige Schicht aus grauen, deutlich gröberen Kiesen, die mit kaum gerundeten Blöcken aus Buntsandstein- und Tertiärquarzit einsetzt. Radiometrische Datierungen von Muddehorizonten, die in diese grauen Kiese eingeschaltet waren, ergaben ${ }^{14} \mathrm{C}$ Alter zwischen 40.000 und 30.000 BP (Kiesgruben 


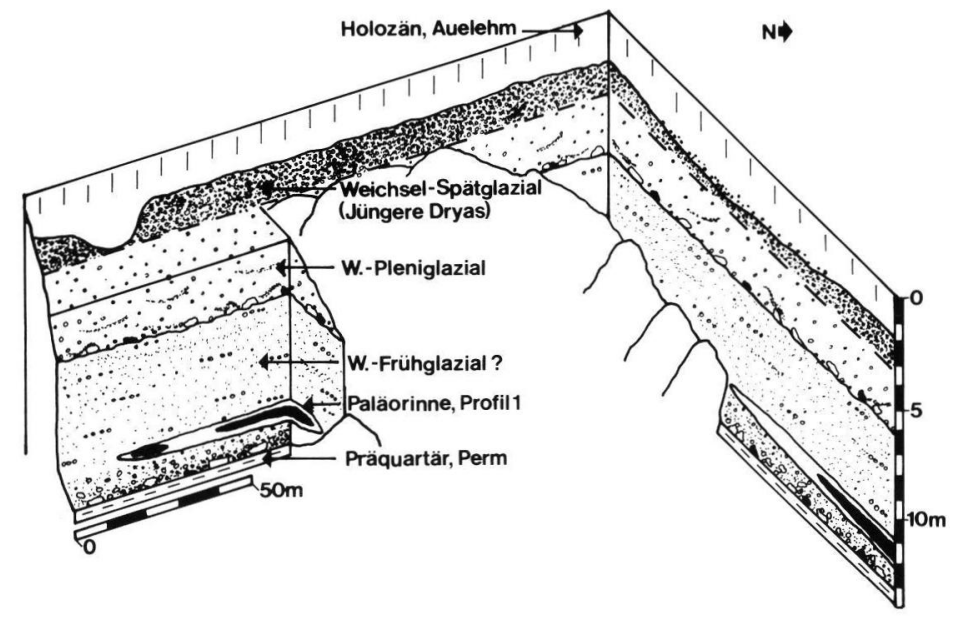

Abb. 2: Stratigraphische Sedimentabfolge der Niederterrasse im südwestlichen Teil der Kiesgrube Weimar-Niederweimar I (Abbau 1991-1993) mit Profil I (aus URz 1995, verändert).

Fig. 2: Stratigraphy of the Niederterrasse in the south-western part of the gravel pit Weimar-Niederweimar I (quarried 1991-1993) with profile I (adapted from URz 1995).

Niederweimar und Roth, siehe Urz 1995). Die Datierungen belegen eine Akkumulation dieses Schotterkörpers während des Weichsel-Pleniglazials. Mit Beginn des Weichsel-Spätglazials und der Umstellung des Gerinnebettmusters zum mäandrierenden Fluss setzte auf den Schottern der Niederterrasse die Ablagerung von Hochflutlehm und eine weitere Verlandung von PaläoRinnen ein. Fluvial umgelagerte Tephra der Laacher See-Eruption nivellierte gegen Ende des Alleröd-Interstadials das noch vorhandene Talbodenrelief weitgehend. Eine verstärkte Flussdynamik zu Beginn der Jüngeren Dryas hatte erneute Schotterumlagerungen und die Akkumulation der Jüngeren Niederterrasse zur Folge. Den Abschluss der Sedimentation im Niederterrassenbereich bilden schließlich Auelehme der Jüngeren Dryas und des Holozäns.

\section{Lithostratigraphischer Aufbau der Profile I-IV}

In der Kiesgrube Weimar-Niederweimar wurden nahe der Quartärbasis vier Profile aus verlandeten Paläo-Rinnen gewonnen und unter stratigraphischen, pollen- und makrorestanalytischen Fragestellungen bearbeitet (zur Lage siehe Abb. 1). Die Profile " aren durch die Kiesgewinnung im Trockenabbau jeweils für kurze Zeit zugänglich und wurden im Block geborgen.

Die zwischen $8 \mathrm{~m}$ und $9 \mathrm{~m}$ unter der heutigen Geländeoberfläche in Schotter und Sand einge- tieften Rinnen sind parallel zum Talverlauf N-S-gerichtet. Die Sedimentmächtigkeiten in diesen Altläufen betragen in der Regel nicht mehr als $1 \mathrm{~m}$, so dass die Gewässer relativ flach gewesen sein dürften. Profil I zeigt an der Rinnenbasis eine Schluffmudde, über der sich ein geringmächtiger Niedermoortorf ausgebildet hat. Über dem Torf folgt ein grauer, feinsandiger Lehm, der darauf hinweist, dass der verlandete Altlauf schließlich von einem Hochflutlehm bedeckt wurde. Die Pollenprofile II-IV wurden aus unterschiedlichen Anschnitten einer weiteren Paläo-Rinne entnommen, die komplett mit sandigen Schluff- und Detritusmudden verfüllt war. Hier kann von einer raschen Sedimentation der Mudden ausgegangen werden.

Profil I (TK 25, 5218, R 3481380, H 5624630 , $177 \mathrm{~m}$ ü. NN)

o $-2 \mathrm{~m}$ u. GOF Auelehm, Holozän

-5 sandige Schotter, grau, Pleni- und Spätglazial

-........ . . Grobblocklage

-9,50 Sande und Schotter, gelblich

-9,61 Lehm, feinsandig (Pollenprofil I)

-9,65 Niedermoortorf, stark komprimiert (Pollenprofil I)

$-9,83$ Schluffmudde, grau, an der Basis sandiger (Pollenprofil I)

- 10 Feinsand, lehmig, gelblich

- 11 Sockelschotter, z. T. mit gröberen Geröllen und Blöcken

rotviolette Arkosen und Konglomerate, Perm

Profile II-III (TK 25, 5218, R 3481490, H 5624000 , $173,5 \mathrm{~m}$ ü. NN)

$0 \quad-2,5 \mathrm{~m} \mathrm{u}$. GOF Auelehm, Holozän

$-2,8$ Sand/Mudde-Wechsellagen, Frübholozän

- 5,5 sandige Schotter, Pleni- und Spätglazial

. . . . . . . . . Grobblocklage

-8 sandige Schotter, gelblich

$-8,3$ kompakte Sande, gelblichbraun

8,3-8,8 Schluffmudde, feinsandig, grau

(Pollenprofil II)

8,3-9,10 Schluffmudde, feinsandig, grau (Pollenprofil III)

- 10 Sockelschotter, grob 
Profil IV (TK 25, 5218, R 3481520, H 5624280 ,

$174,5 \mathrm{~m}$ ü. NN)

$0 \quad-8,5 \mathrm{~m}$ u. GOF Sedimente durch den Kiesabbau abgedeckt

-8,6 Feinsand, schluffig-lehmig, rostfarbig-beige

-8,88 Grobdetritusmudde, humos, feinsandig, dunkelbraun, Nadelhölzer

\section{(Pollenprofil IV)}

-9,12 Mudde, schluffiger Lehm, grau, (Pollenprofil IV)

-9,20 Feinsand, schluffig-lehmig, gelblich

- 10 Sockelschotter

rotviolette Arkosen über kieseligen Tonschiefern, Perm über Unterkarbon

Für die Analyse botanischer Makroreste standen von Profil I 6 Proben der im Block geborgenen Profilsäule zur Verfügung, wobei jeweils zwischen 0,9-1 1 Sediment pro Probe aufgearbeitet wurden. Den Profilen II-IV wurden aus frischen Anschnitten jeweils ca. 101 Mudde entnommen. Die Proben wurden in Wasser eingeweicht und mit einen Siebsatz von $0,63 \mathrm{~mm}, 0,31 \mathrm{~mm}$ und $0,18 \mathrm{~mm}$ Maschenweite geschlämmt. Aus dem getrockneten Material ließen sich mit einem Stereomikroskop die botanischen Großreste bei 10-30facher Vergrößerung auslesen. Die Bestimmung erfolgte über einschlägige Bestimmungsliteratur und den Vergleich mit Material der eigenen Großrestsammlung.

Die Ergebnisse der Auswertung von Profil I wurden in Anlehnung an die Darstellungsweise von Pollendiagrammen mit Hilfe der EDV-Programme TILIA und TILIA-graph (GRImm 1992) als Großrestdiagramm dargestellt (Abb. 4). Die YAchse zeigt die beprobten Horizonte, ihre Tiefe und das jeweilige Substrat. Die X-Achse gibt die Anteile der unterschiedlichen Taxa in absoluten Zahlen wieder. Die Gesamtsumme enthält alle im Diagramm aufgeführten botanischen Großreste inklusive sonstiger Reste. Die Zonierung richtet sich nach der Unterteilung des entsprechenden Pollendiagramms.

Da die Profile II-IV nach den Ergebnissen der Pollenanalyse nur jeweils relativ kurze, einheitliche Kiefern-Fichten-dominierte Phasen der Vegetationsentwicklung widerspiegeln, wurde nur jeweils eine botanische Großrestprobe pro Profil ausgewertet. Die Darstellung erfolgt in Form von Balkendiagrammen, wobei die Großreste der Profile II-IV getrennt nach ökologischen Gruppen (Gehölze, Feuchtgrünland und Schlammufer, Röhricht und Seggenried) aufgeführt werden. Ein Kreisdiagramm (Profil II) zeigt schließlich beispielhaft die heutigen geographischen Verbrei- tungsgebiete nachgewiesener Pflanzenarten. Die Angaben dazu wurden Oberdorfer (1990) entnommen.

Die Aufbereitung für die Pollenanalyse folgte der Standard-Methode nach ERDTMAn, wobei jedoch auf eine Acetolyse verzichtet wurde. Die Auszählung erfolgte auf eine Summe von ca. 500 Gesamtpollen, die auch die Berechnungsgrundlage dargestellter Diagramme bilden.

\section{Ergebnisse der pollen- und makrorest- analytischen Untersuchungen}

Sowohl das Pollendiagramm als auch das Makrorestdiagramm des Profiles I lassen sich biostratigraphisch in vier deutlich voneinander getrennte Biozonen unterteilen (Abb. 3, 4). Im Pollendiagramm treten an der Basis vor allem krautige Arten auf, unter denen Liguli- und Tubuliflorae, $A r$ temisia und Thalictrum hervorzuheben sind. Besonders das Vorkommen letzterer Gattungen deutet auf eine noch lichte Vegetation hin. Der Birkenpollenanteil liegt mit nahezu 50\% schon relativ hoch. Wie die Makrorestanalyse zeigt, kamen als Pollenspender sowohl Baumbirken (Betula "alba"-Gruppe) als auch Zwergbirken (Betula nana) in Betracht. Abb. 4 verdeutlicht weiterhin, dass ein aus verschiedenen Sauergräsern und dem Pfeilkraut (Sagittaria sagittifolia) aufgebauter Röhrichtgürtel das Gewässer säumte. Das zahlreiche Auftreten von Cristatella mucedoStatoblasten und Charophyten-Oogonien ist hierbei Indiz für einen klaren Wasserkörper. Obwohl die Zone nur eine Probe umfasst, wird sie aufgrund oben erwähnter Unterschiede zur nachfolgenden LPAZ als eigener Abschnitt ausgegliedert.

Der Beginn der nächsten Biozone zeichnet sich sedimentologisch nicht ab; die Schluffmudde bleibt weiterhin erhalten. Im Pollenspektrum geht der Anteil typischer Heliophyten bei gleichzeitig ansteigendem Baumpollenanteil deutlich zurück. Die Bewaldung erfolgte hauptsächlich durch Birken, wobei jetzt nur noch Reste von Baumbirken gefunden wurden (Abb. 4). Palynologisch konnten als neue Arten Lärche und auch Eiche und Ulme nachgewiesen werden. Makrorestanalytisch ist nur Larix vertreten, so dass ein autochthones Vorkommen als gesichert angesehen werden kann. Die Lokalvegetation im Uferbereich war weiterhin durch einen Röhrichtgürtel geprägt, allerdings breitete sich im Gewässer nun verstärkt ein Laichkrautgürtel aus, an dem neben verschiedenen Potamogeton-Arten auch Krebsschere (Stratiotes spec.) und Wasserhahnenfuß (Ramunculus aquatilis s. 1.) beteiligt waren. Es 
Kiesgrube Weimar-Niederweimar

Profill

Höhe NN $+177 \mathrm{~m}$

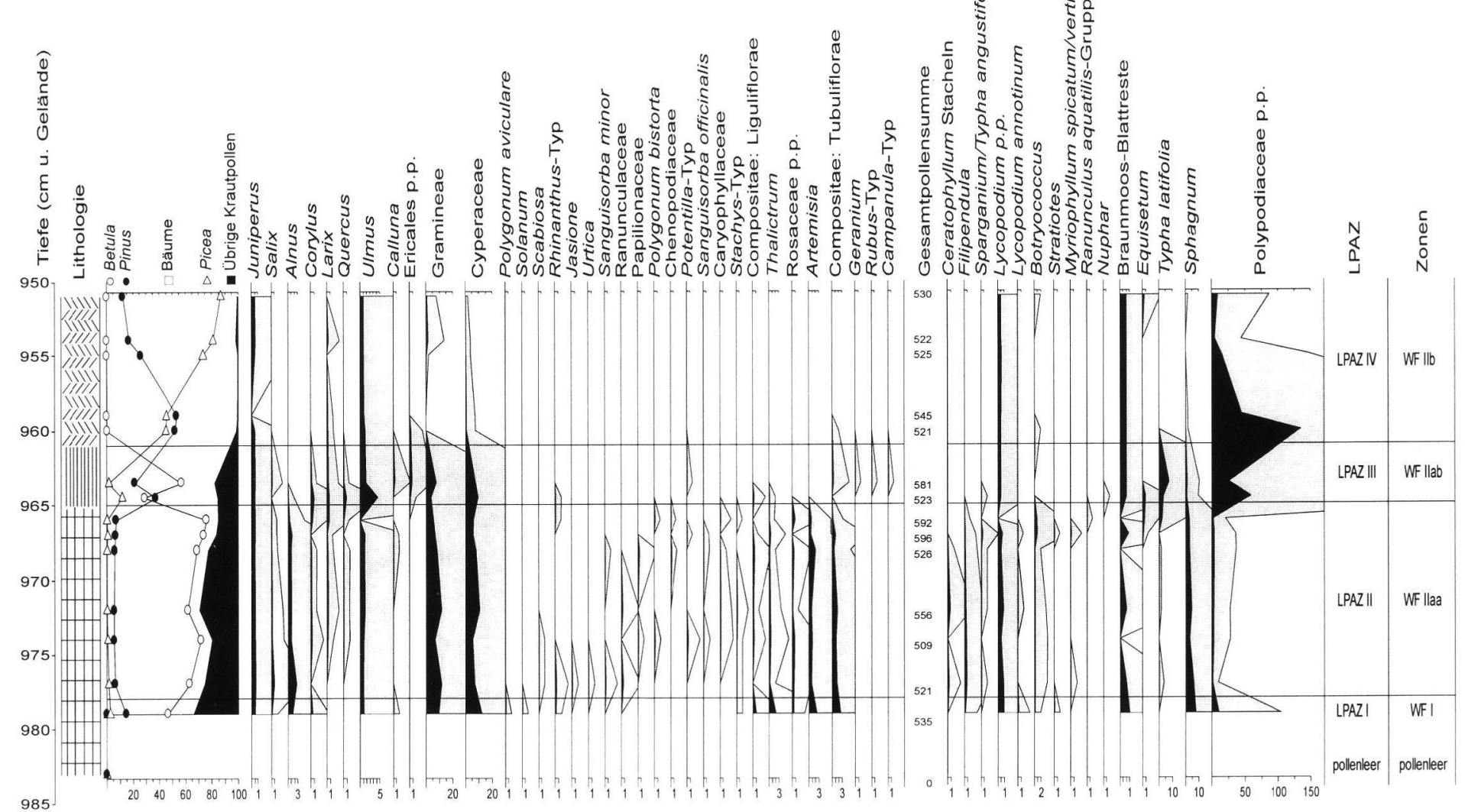

Abb. 3: Vereinfachtes Pollendiagramm des Profiles I aus der Kiesgrube Weimar-Niederweimar. +/++ kennzeichnen ein einfaches, bzw. mehrfaches Vorkommen außerhalb der Zählung, die gerasterte Fläche markiert eine 10fache Überhöhung des Kurvenverlaufs. Die Zonierung des Diagrammes erfolgt in LPAZ (local pollen assemblage zones) und nach CASPERS \& Freund (1997).

Fig. 3: Simplified pollen diagram of profile I, gravel pit Weimar-Niederweimar. ' + ' and '++' denote a single record and several records, respectively, of a pollen taxon noted outside the pollen count. Silhouette curves show pollen representation exaggerated x10. Local pollen assemblage zones are indicated and the nomenclature as in CASPERS \& FREUND (1997). 


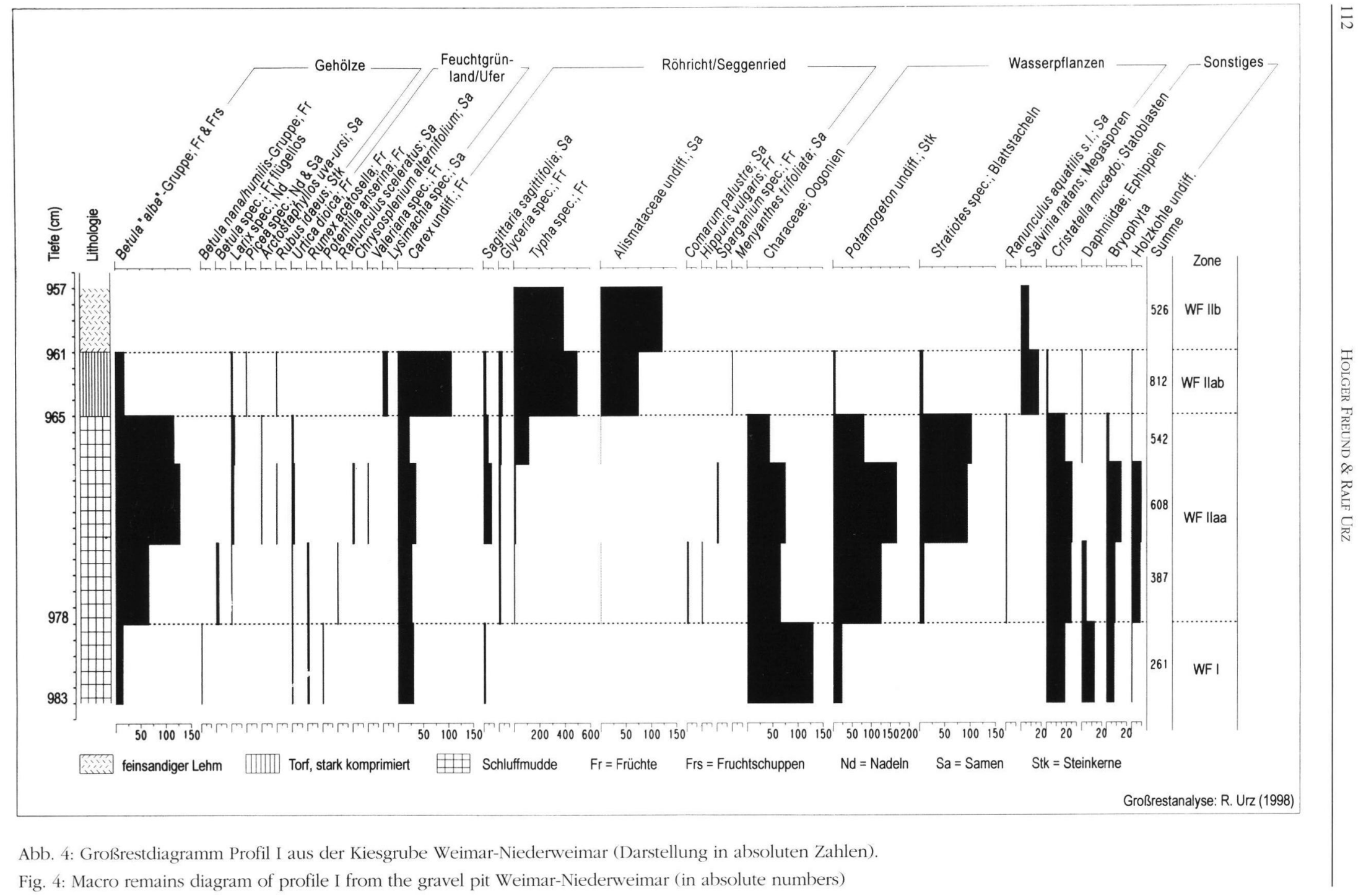


muss sich um ein basen- und nährstoffreiches, stehendes oder aber nur sehr schwach fließendes Gewässer gehandelt haben. Bemerkenswert ist weiterhin der Nachweis von Ceratophyllum-Blattstacheln, was ebenso wie das Auftreten von Stratiotes spec. auf ein relativ sommerwarmes Klima schließen lässt (Abb. 3).

Mit dem Sedimentwechsel bei $965 \mathrm{~cm}$ unter Geländeoberfläche ist auch im Pollendiagramm eine deutliche Änderung angezeigt. Die Birkendominanz wird durch eine Phase mit wechselnden Kiefern- und Birkenanteilen gebrochen. Auch die Fichte war mit Sicherheit am Vegetationsaufbau beteiligt, was durch Fichtennadel-Funde im Torf belegt ist. Auffallend sind auch die sehr hohen Ulmenwerte von nahezu 5\%, so dass ein Aufwachsen von Ulmus im Auenbereich wahrscheinlich war. Die Lokalflora zeigt eine Verlandung, an der, neben verschiedenen Seggen (Carex undiff. mit Carex rostrata/vesicaria-Typ), Rohrkolben (Typha spec., Typha latifolia) und Froschlöffelgewächsen (Alismataceae), auch Fieberklee (Menyanthes trifoliata) und Schwimmfarn (Salvinia natans) beteiligt waren.

Die letzte Biozone, die sich sedimentologisch als feinsandiger Lehm abzeichnet, ist in besonderem Maße durch eine Zersetzungsauslese gekennzeichnet. Der Lehm entspricht möglicherweise einem in Rinnenposition abgelagerten Hochflutlehm. Das Pollenspektrum ist sehr artenarm und wird durch Pollenkörner der Kiefer und Fichte sowie durch perisporlose monolete Sporen (Polypodiaceae) dominiert. Diese reichern sich sehr häufig durch eine Zersetzungsauslese im Pollensubstrat an. Zu Beginn und gegen Ende dieser Pollenzone treten auch Pollenkörner von Lärche und Wacholder auf. Diese sind in der Regel leicht korrodierbar. Inwieweit es sich hier um Umlagerungen bzw. Einschwemmungen mit kurzem Transportweg handelt, muss an dieser Stelle offen bleiben. Als einzige pflanz-

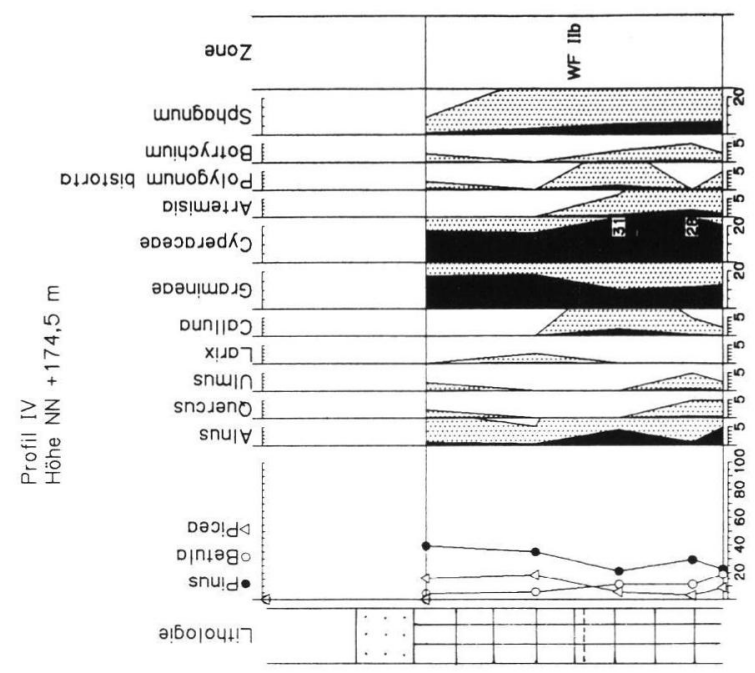




\section{Gehölze:}

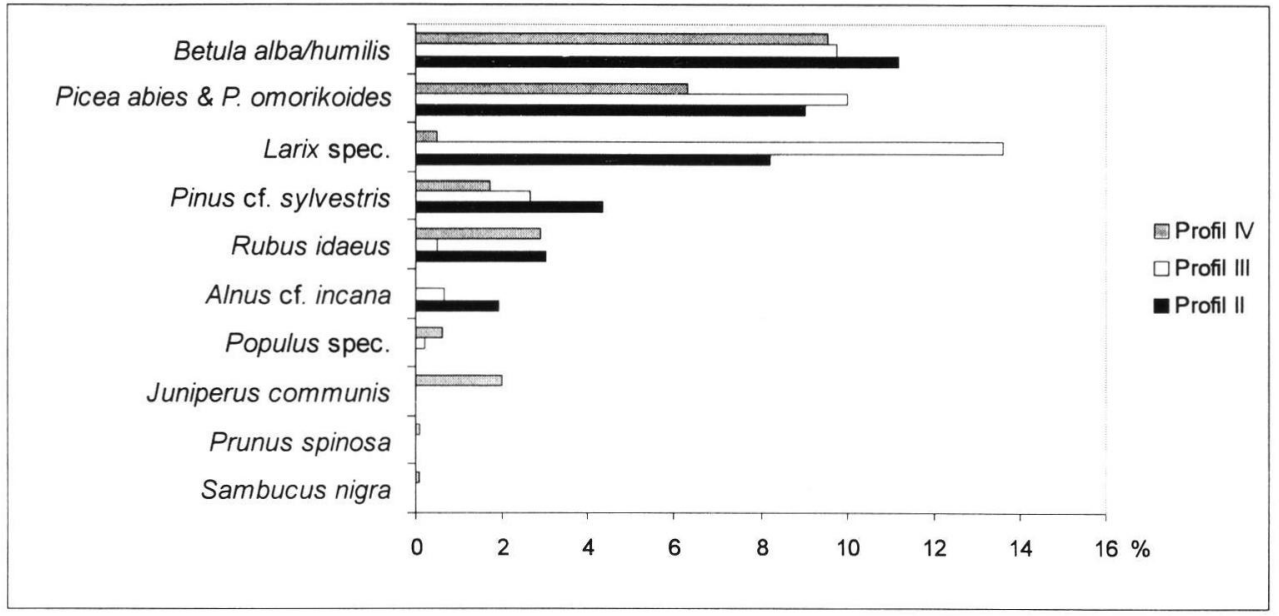

\section{Feuchtgrünland und Schlammufer:}

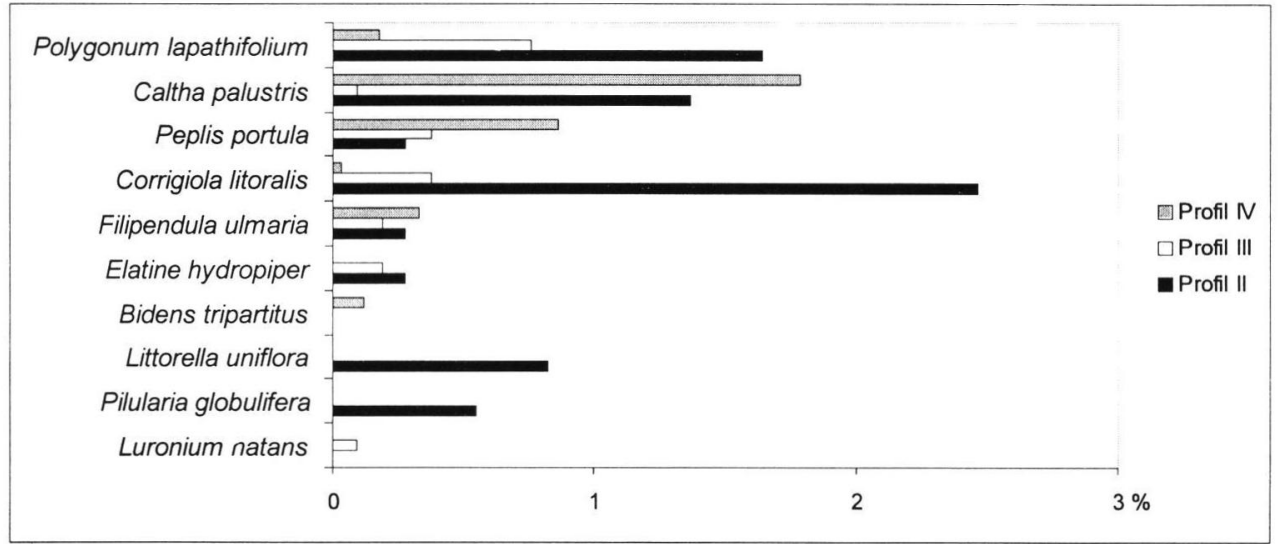

\section{Röhricht und Seggenried:}

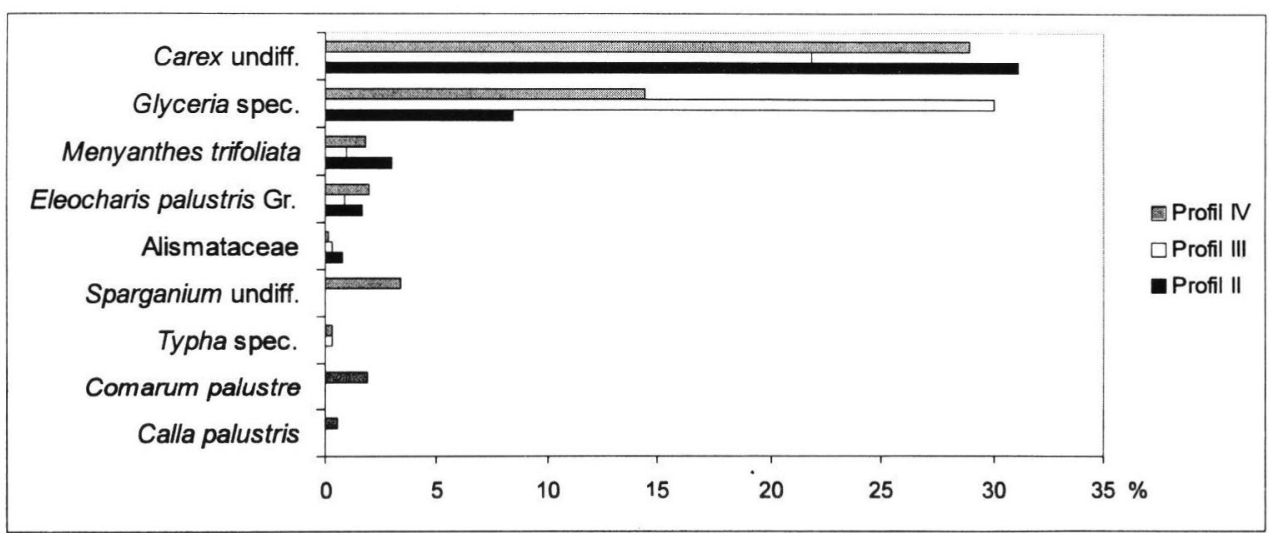

Abb. 6: Großreste ausgewählter ökologischer Gruppen und ihr Vorkommen innerhalb der Profile Niederweimar II-IV. Die Balkendiagramme zeigen die prozentualen Anteile der aufgeführten Taxa an der Gesamtsumme der nachgewiesenen Großreste aus einem Profil.

Fig. 6: Macro remains of selected ecological groups and their presence within the profiles Niederweimar II-IV. The bar charts show for each taxon mentioned the percentage of the total amount. 
liche Großreste waren, neben Makrosporen vom Schwimmfarn (Salvinia natans), nur noch die Früchte von Rohrkolben (Typha spec.) und Froschlöffelgewächsen (Alismataceae undiff.) vertreten. Deren Großreste sind zwar sehr widerstandsfähig, trotzdem waren sie hier teilweise bis zur Unkenntlichkeit korrodiert.

Um den biostratigraphisch wichtigen Übergang Birken/KiefernPhase in die Kiefern-Fichtenphase besser fassen zu können, wurden drei weitere Profile aufgenommen und in ähnlicher Weise wie Profil Niederweimar I untersucht (Niederweimar II-IV, Abb. 5).

Alle drei Diagramme zeigen im Pollendiagramm eine eindeutige Kiefern-Dominanz mit einem mehr oder minder hohen Anteil an Fichte, der um $20 \%$ schwankt. Durch starkes Auftreten von Pollenkörnern der Lokalvegetation an der Gesamtpollensumme, hier vor allem Gräser (Gramineae) und Sauergräser (Cypcraceae), ist der Baumpollen-Anteil im Profil Niederweimar IV rechnerisch unterrepräsentiert. Alle drei Diagramme zeigen nur eine geringe Sukzession, so dass von einer schnellen Auffüllung der Rinnen ausgegangen werden muss.

Die Profile Niederweimar II-IV sind auch durch gleichartige Großrest-Spektren gekennzeichnet. Sie unterscheiden sich zwar in den mengenmäßigen Anteilen einzelner Taxa, das Artenspektrum ist aber in der Regel ähnlich (Abb. 6). Hauptbaumarten sind demnach Baumbirke, Fichte, Kiefer, Lärche, Grau-Erle (Alnus cf. incana) und zum Teil auch Pappel (Populus spec.) und Wacholder (Juniperus communis). Über Sand und Schotter könnte sich also eine fichtenreiche GrauerlenAue entwickelt haben. Dieser Auwald mit boreal-hochmontanem Charakter ist auch heute optimal

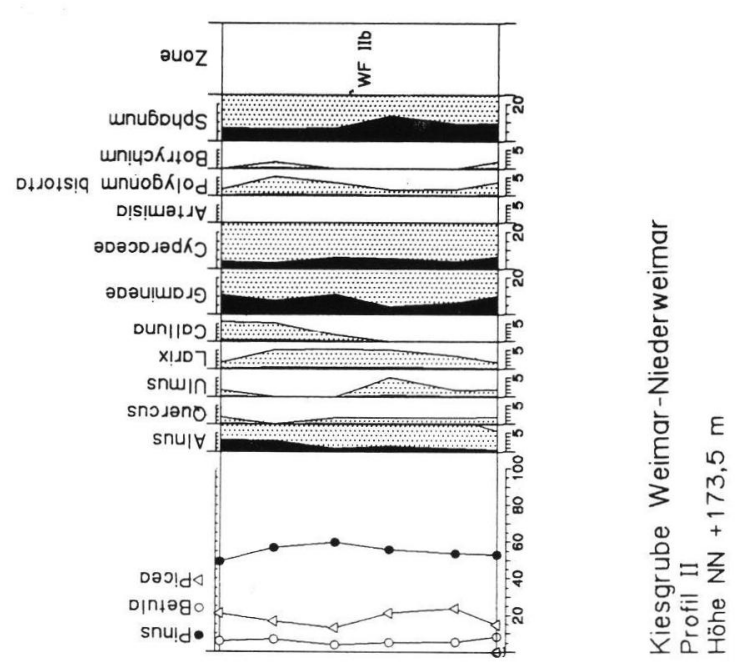

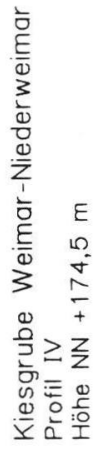

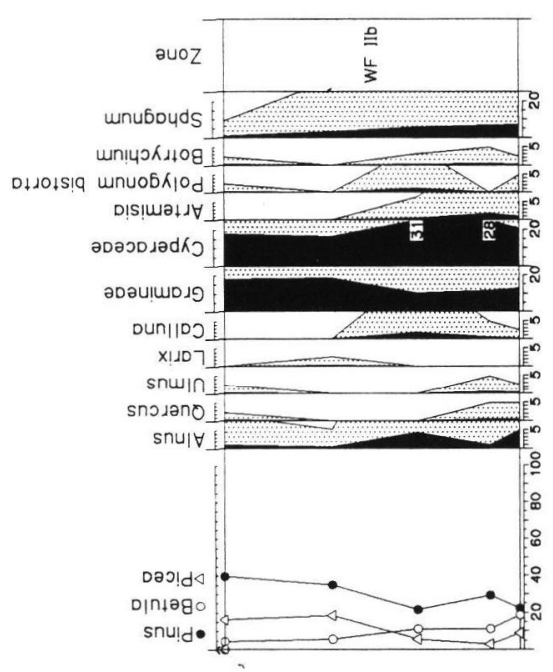


im potentiellen Nadelwaldgürtel der Gebirge und Mittelgebirge vertreten (OBERDORFER 1992, POTT 1995: 557f). Nach den palynologischen Ergebnissen waren auch Ulme und Eiche am Waldaufbau beteiligt. Dass die Kiefer im Makrorestdiagramm hinter der Fichte zurückbleibt, im Pollendiagramm aber dominiert, liegt vermutlich daran, dass die Fichte bevorzugt feuchtere Standorte besiedelte. Ihre Wuchsorte lagen somit näher an den Profilstellen als die der Kiefer, die auf trockeneren Standorten zusammen mit Wacholder dominierte. Weiterhin wurden als anspruchsvolle, wärmeliebende Gehölze Schlehe (Prunus spinosa) und Schwarzer Holunder (Sambucus nigra) nachgewiesen. Auch bei den Wasserpflanzen ist der Anteil wärmeliebender Elemente besonders hoch. Wie im Profil Niederweimar I wurden sowohl Salvinia-Megasporen als auch Großreste der Krebsschere (Stratiotes-Blattstacheln) gefunden. Im Röhricht und Seggenried dominierten erneut verschiedene Seggen-Arten und Wasserschwaden (Glyceria spec.); Fieberklee (Menyanthes trifoliata) war ebenfalls häufig vertreten.

Besonders wichtig für die spätere Diskussion der klimatischen Verhältnisse ist der Nachweis von Pflanzen der Schlammufervegetation und des Feuchtgrünlandes. Mit Arten wie dem Sumpfquendel (Peplis portula), dem Hirschsprung (Corrigiola litoralis), dem Strandling (Littorella uniflora), dem Pillenfarn (Pilularia globulifera) oder dem Froschkraut (Luronium natans) finden sich hier viele Vertreter mit ausgeprägtem subatlantischem Verbreitungsschwerpunkt (Abb. 6, Tafel 1).

Generell zeigt sich, dass die Profile Niederweimar II-IV zeitgleich sind, da sie sich sowohl palynologisch als auch makrorestanalytisch gut parallelisieren lassen (Abb. 6). Leider weisen sie keine eindeutige Überschneidung mit dem Profil Niederweimar I auf, eine mögliche Konnektierung zeigt Abb. 7. Da im Profil Niederweimar II die Birken-Kiefer-Phase fehlt, würde das Profil II bereits in der von Kiefern-Pollenkörnern dominierten LPAZ IV des Profiles I ansetzen. Ulme und Lärche sind, wie im Profil I, im Pollendiagramm vertreten, wohingegen die Fichtenkurve schwächer ausgebildet ist. Dies ist, wie bereits erwähnt, durch eine Zersetzungsauslese erklärbar. Es wird ebenfalls deutlich, dass nicht der komplette Verlauf dieser klimatisch günstigen Phase (Interstadial oder Interglazial) überliefert ist. Die Profile Niederweimar II-IV brechen allesamt innerhalb der Kiefernphase ab, so dass der Übergang in das nächste Stadial/Glazial nicht erfasst ist.

\section{Datierung, überregionale Einordnung und paläoklimatische Relevanz der Profile Niederweimar I-IV}

Bei der biostratigraphischen Einordnung und Gliederung der Profile Niederweimar I-IV ist zu klären, ob es sich um ein ausgehendes Interglazial (Eem-Interglazial) oder um ein weichselzeitliches Interstadial handelt. Eine Datierung in noch ältere Abschnitte des Quartärs ist sowohl in geologischer als auch botanischer Hinsicht unwahrscheinlich. Erste Hinweise auf eemzeitliche Ablagerungen in Terrassenschottern der mittleren Lahn stammen von Huckriede (1972). Aus der Kiesgrube Niederweimar wurden ca. 1,2 m über der Quartärbasis botanische Makroreste aus Mudden und Seggen-Torfen ausgewertet. Es konnten u. a. Picea abies, Carpinus betulus, Corylus avellana, Quercus spec., Betula pubescens, Alnus glutinosa, Prunus divaricata/ cerasifera-Gruppe, Salvinia natans, Stratiotes aloides und Ceratophyllum demersum nachgewiesen werden. Für eine vorläufige Einstufung zwischen Hainbuchen-Zeit und Kiefern-Fichten-Tannen-Zeit des Eem durch HuCKRIEDE (1972) sprach vor allem der hoh'e Anteil klimatisch anspruchsvoller Gehölze sowie die Abwesenheit von Pinus und Picea omorika/ omorikoides.

Erläuterung zu Tafel 1:

1: Larix spec., Lärche, Nadel, Maßstab $300 \mu \mathrm{m}$

2: Betula "alba"-Gruppe, Baumbirke, Fruchtschuppe, Maßstab $1000 \mu \mathrm{m}$

3: Alnus incana (L.) MoEnch, Grau-Erle; Frucht, Maßstab $1000 \mu \mathrm{m}$

4: Polygonum lapatbifolium L., Ampfer-Knöterich, Frucht, Maßstab $1000 \mu \mathrm{m}$

5: Rubus idaeus L., Himbeere, Steinkern, Maßstab $300 \mu \mathrm{m}$

6: Scleranthus cf. annuus-Gruppe, Knäuelkraut, Kelch, Maßstab $1000 \mu \mathrm{m}$

7: Stratiotes spec., Krebsschere, Blattstachel, Maßstab $300 \mu \mathrm{m}$

8: Elatine hydropiper L., Wasserpfeffer-Tännel, Same, Maßstab $100 \mu \mathrm{m}$

9: Corrigiola litoralis L., Hirschsprung, Frucht, Maßstab $300 \mu \mathrm{m}$

10: Luronium natans (L.) RAF., Froschkraut, Achäne, Maßstab $300 \mu \mathrm{m}$

11: Peplis portula L., Sumpfquendel, Same, Maßstab $300 \mu \mathrm{m}$

12: Littorella uniflora (L.) Aschers., Strandling, Frucht, Maßstab $300 \mu \mathrm{m}$ 
Taf. 1: Florenelemente des Brörup-Interstadials von Weimar-Niederweimar, Profile I-IV (Aufnahmen: Dr. A. SCHAPER, Fachbereich Geowissenschaften der Philipps-Universität Marburg).

Plate 1: Floral elements of the Brörup Interstadial from Weimar-Niederweimar, profile I-IV (Photos by Dr. A. SCHAPER, Faculty of Geosciences, Philipps-Universität Marburg)
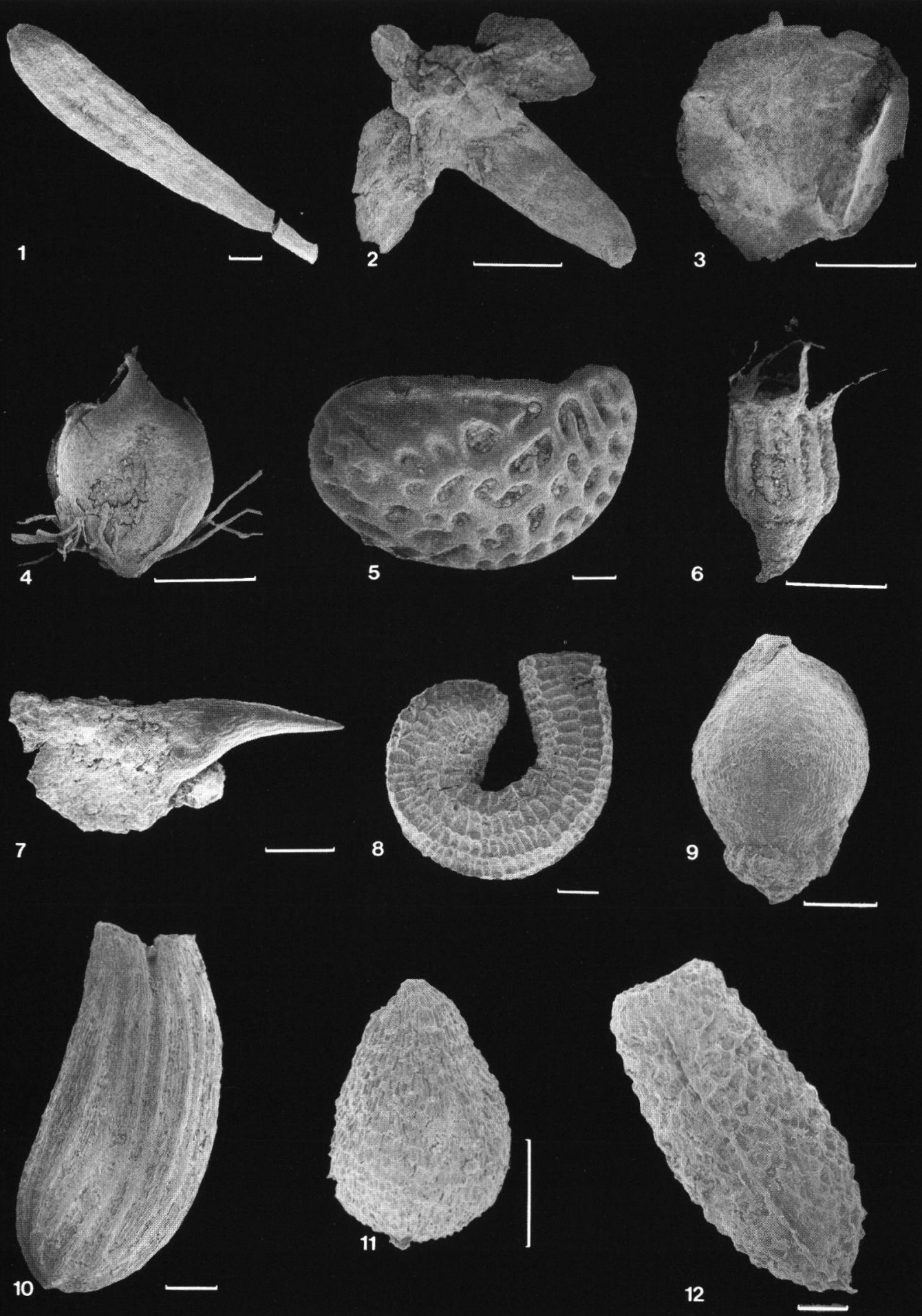
Nach den Ergebnissen der vorliegenden Pollenund Großrestanalyse beginnt die Vegetationsentwicklung im Profil Niederweimar I zunächst mit einer offenen Vegetation mit Heliophyten und auch Zwerg-Birken. Eine derartig offene Vegetation ist für das ausgehende Eem-Interglazial (Pollenzonen E VI/VII nach Menke \& TYNNI 1984) bislang nicht belegt. Eine spät-eemzeitliche Kältephase müsste aufgrund von Erosion auch zu Sedimentverlagerungen führen, so dass mit einem deutlich höheren Anteil umgelagerter Pollenkörner von Fichte, Tanne, und anderer wärmeliebender Taxa zu rechnen wäre. Dies ist, wie Abb. 3 zeigt, eindeutig nicht der Fall.

Biostratigraphisch interessant ist vor allem das Auftreten der Lärche, da ihr Vorkommen als Charakteristikum frühweichselzeitlicher Ablagerungen angesehen wird (Abb. 3, 4 sowie Taf. 1: 2). Eemzeitliche Larix-Pollenfunde sind zwar vereinzelt aus Ost- und Süddeutschland (GRÜGER 1979: Riss/Würm-Interglazial Zonen DA 10-12 mit Larix, ERD 1973: Profil Kittlitz, Einzelfunde in den Zonen E 8/9) beschrieben, in West- und Norddeutschland sind sie aber selten. Auch die im Profil Niederweimar I durch Großreste nachgewiesene Serbische Fichte (Picea omorika oder P. omorikoides) tritt im Jungquartär Mitteleuropas vor allem in den Frühweichsel-Interstadialen auf, so dass auch dieser Befund eindeutig gegen ein eemzeitliches Alter spricht. Bei der Beurteilung, ob es sich im vorliegenden Pollendiagramm um das Brörup- oder Odderade-Interstadial handelt, ist das Einwanderungs- und Ausbreitungsverhalten von Fichte und Lärche ausschlaggebend. Im Odderade-Interstadial wanderten Fichte und Lärche erst in der Kiefernphase ein, wobei Picea stets vor Larix zu verzeichnen ist. Im Brörup-Interstadial sind beide Bäume schon in der Birkenphase vertreten, die Reihenfolge der Einwanderung ist regional unterschiedlich. So wanderte im subatlantischen Bereich Picea vor Larix ein, im subkontinentalen Bereich geschah dies umgekehrt oder gleichzeitig. Im Pollendiagramm Weimar-Niederweimar sind beide Arten schon in der Birkenzone nachweisbar. Eine chrono- und biostratigraphische Einordnung des Profiles Niederweimar I in das Brörup-Interstadial ist demnach wahrscheinlich. Die Basislage wäre demzufolge dem Herning-Stadial (LPAZ I = Pollenzone WF I nach Menke \& TYnn 1984), die ausgeprägte Birkenphase (LPAZ II) der Pollenzone WF IIaa (nach CASPERs \& Freund 1997) des Brörup-Interstadials zuzuordnen. Der Wechsel von birken- zu kieferndominierten Wäldern im Brörup-Interstadial ist oft durch einen Übergang gekennzeichnet, in dem Betula und Pinus miteinander um die Vorherrschaft konkurrierten. Die Lokalpollenzone III des Profils Niederweimar I entspricht diesem Zustand der Waldentwicklung und ist nach CASPERS \& Freund (1997: 213ff) in die Pollenzone WF IIab zu stellen. Sowohl die Diagramme Niederweimar II-IV als auch der obere Abschnitt des Profiles Niederweimar I (LPAZ IV) entsprechen dem kiefernreichen Abschnitt des ersten frühweichselzeitlichen Interstadials (WF IIb). Aufgrund von Zersetzungsauslese oder der zu geringen zeitlichen Auflösung lässt sich eine weitergehende Untergliederung dieses Abschnittes nicht vornehmen.

Aufgrund ihres brörupzeitlichen Alters sind die Profile aus der Grube Weimar-Niederweimar überregional bedeutend, da sie eines der wenigen bekannten Bindeglieder zwischen den frühweichselzeitlichen Untersuchungspunkten des nördlichen Mitteleuropas und des Alpenvorlandes darstellen. Abb. 8 zeigt einen Vergleich dreier Pollendiagramme: Groß Todtshorn aus Niedersachsen (CASPERS 1997), Samerberg aus Oberbayern (GRÜGER 1979) und Niederweimar in Hessen. Die Ähnlichkeiten zwischen den Profilen der mittleren Lahn und dem norddeutschen Tiefland sind augenscheinlich; das Profil Samerberg unterscheidet sich dagegen deutlich. Ursachen hierfür sind sowohl die Einwanderung der Arten als auch die Höhenlage des Profils Samerberg von ca. $\mathrm{NN}+600 \mathrm{~m}$ gegenüber ca. $\mathrm{NN}+175 \mathrm{~m}$ in Niederweimar.

Groß Todtshorn und Niederweimar zeigen beide eine deutliche Birkenphase, Birken-Kiefernphase und eine anschließende Kiefernphase, wobei in Niederweimar der Fichtenanteil, wie die Profile Niederweimar II-IV (Abb. 5) zeigen, etwas höher liegt als im norddeutschen Tiefland. Während in Norddeutschland als Laubbäume nur Birken und Erlen vorkamen, ist an der mittleren Lahn auch die Ulme heimisch. Dies unterstützt die Argumentation von BeHRE (1974), der darauf hinwies, dass die nördliche Verbreitungsgrenze verschiedener Laubgehölze ungefähr an der Mainlinie gelegen haben müsste. Es ist nicht verwunderlich, dass es sich bei den gefundenen Laubhölzern in der Mehrzahl um Arten der Auewälder handelt. Ulme, Pappel, Grau-Erle, und vermutlich auch die Eiche sind, von Süden kommend, entlang der großen Flussläufe nach Norden vorgestoßen. Da die Länge des Brörup-Interstadials ausreichend für eine ausgedehnte nördliche Migration gewesen wäre, muss ihre weitere Ausbreitung infolge 

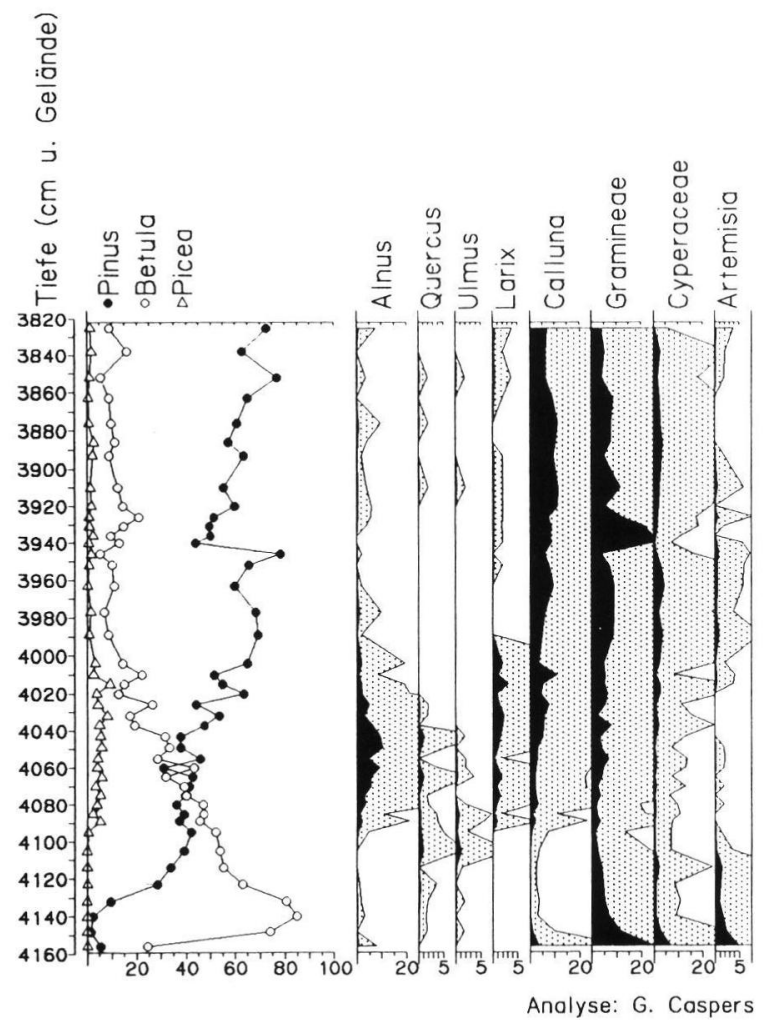

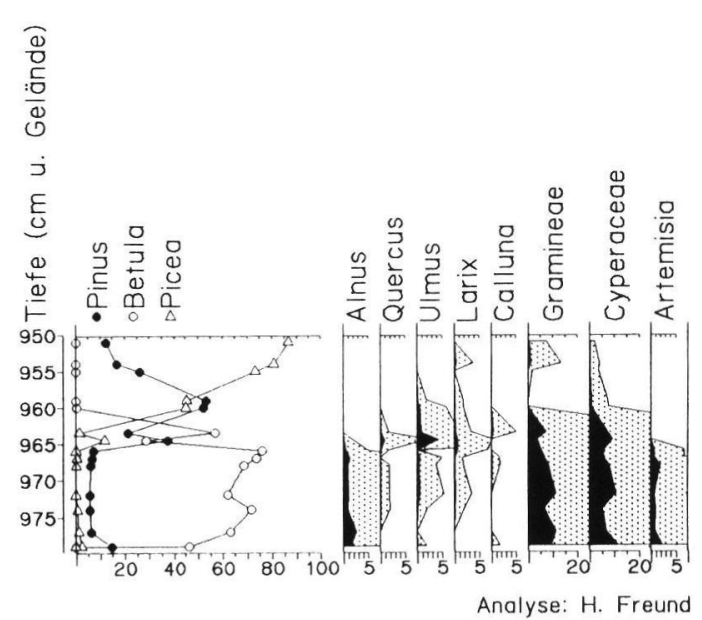

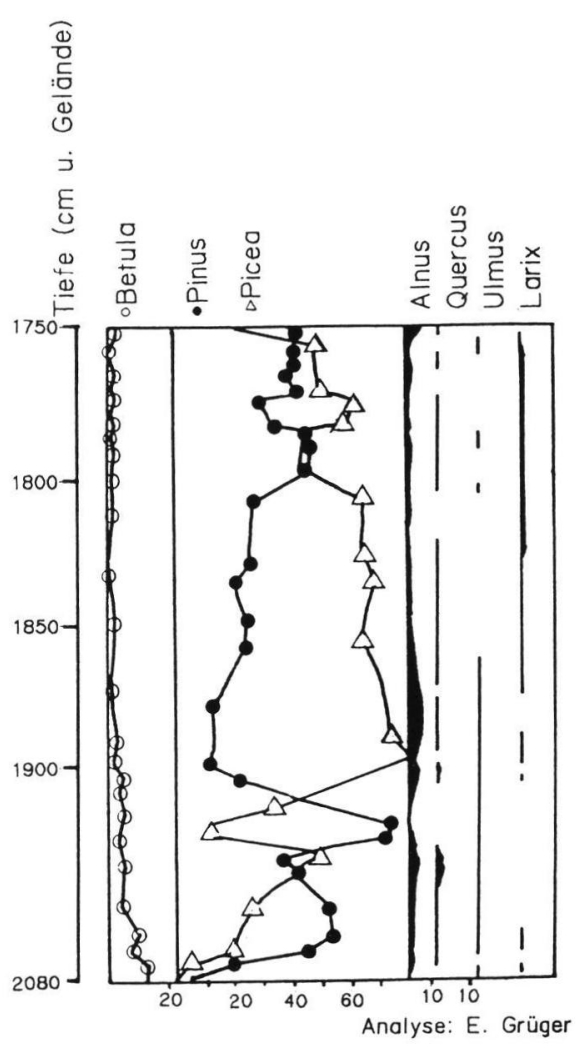

Abb. 8: Überregionaler Vergleich von Pollendiagrammen des Brörup-Interstadials aus Niedersachsen (Groß Todtshorn: CASPERS 1997), Oberbayern (Samerberg: GRÜGER 1979) und dem mittleren Lahntal in Hessen. Bei der Interpretation ist die unterschiedliche Tiefenskalierung der Diagramme zu beachten.

Fig. 8: Regional comparison of selected pollen profiles from central Europe: Brörup interstadial profiles from Lower Saxony (Gross Todtshorn: CASPERS 1997), Upper Bavaria (Samerberg: GRÜGER 1979), and the middle reaches of the river Lahn in Hessen. Note the different depth scales. 
klimatischer Ungunst unterbunden worden sein. Hierbei dürften im westlichen Mitteleuropa weder die Sommer- noch tiefe Wintertemperaturen ausschlaggebend gewesen sein, sondern eher die nach Norden zunehmende Kürze der Vegetationsperiode, vermutlich bedingt durch eine längere Schneebedeckung (CASPERS \& FrEund) 1997 , 2000; WaLKLING 1997).

Interessant ist auch das frühe Auftreten der Lärche gegenüber den nord- und ostdeutschen Profilen. Es ist davon auszugehen, dass neben der von CASPERS \& Freund (1997) aufgezeigten Ost-West gerichteten Einwanderung der Lärche nach Norddeutschland auch ein Süd-Nord gerichteter Einwanderungsweg existierte. Ob es sich dabei um Rückwanderungen aus verschiedenen Refugialgebieten handelte, ist zu vermuten, kann an dieser Stelle aber nicht geklärt werden. In Norddeutschland liegt der Zeitpunkt der maximalen Larix-Ausbreitung zeitgleich oder direkt nach dem brörupzeitlichen Klimarückschlag. In Niederweimar ist dieser chronostratigraphisch wichtige Zeitmarker nicht erfasst.

Wie die Vegetationsentwicklung im kiefernreichen Abschnitt des Brörup-Interstadials im Bereich der südlichen Mittelgebirgsschwelle verlaufen sein könnte, lässt sich andeutungsweise aus Pollenanalysen der Mosbacher Humuszonen im Profil Mainz-Weisenau herauslesen (Bıbus et al. 1996). Die mit dem Brörup-Interstadial parallelisierte Humuszone 1 weist von Beginn an hohe Kiefern- und Fichtenanteile auf, eine ausgeprägte, von Birken dominierte Pollenzone fehlt. Diese ist entweder nicht überliefert oder aber aus lokalklimatischen bzw. sukzessionsbiologischen Gründen im Mainzer Trockenbecken nicht entwickelt. Es ist aber auch zu bedenken, dass es in Lössen, ähnlich dem Hochflutlehm im Profil Niederweimar I, zur Zersetzungsauslese kommen kann, so dass eine lokale Überrepräsentation von KoniferenPollenkörnern im Pollenspektrum möglich ist. Lärche wurde in den Mosbacher Humuszonen nicht notiert. Das Vorkommen der Lärche kann regional und auch lokal sehr stark variieren (s. BeHR: 1974), so dass Larix vielleicht aus diesen Gründen hier nicht gefunden wurde.

Die Kiefern-Fichten-Phase der Profile Niederweimar II-IV (bzw. der lehmige Horizont Nieder1990). weimar I) könnte von der Sukzession, aber nicht chronostratigraphisch, dem unteren Drittel der Mosbacher Humuszone 1 entsprechen. Die weitere Entwicklung würde demzufolge von einer Kiefern-Fichten-Phase in eine Fichten-TannenPhase übergehen. Tatsächlich wurden in den Profilen Niederweimar II-IV vereinzelt Pollenkörner der Tanne nachgewiesen. Eine endgültige Klärung werden nur ein vollständigeres Profil oder weitere einzelne Versatzstücke aus der Grube Weimar-Niederweimar bringen.

Besonders erwähnenswert ist in diesem Zusammenhang eine ältere Untersuchung der mittelpleistozänen Tone von Hösbach bei Aschaffenburg am Main von BAAS (1986). Diese Arbeit stammt schon aus der Mitte dieses Jahrhunderts und umfasst palynologische, großrestanalytische und entymologische Untersuchungen; die Ergebnisse wurden aber erst in den 80er Jahren publiziert. Die Pollendiagramme und Makrorestanalysen entsprechen weitgehend den Ergebnissen aus Niederweimar, so dass kein Zweifel daran besteht, dass es sich in Hösbach nicht um ein mittelpleistozänes Interglazial, sondern um ein frühweichselzeitliches Interstadial handelt. Die von BaAs (1986) als Indizien für ein mittelpleistozänes Alter angeführten Punkte:

\section{- Massenentwicklung Picea omorikoides zusammen mit Picea abies \\ - das Auftreten von Larix spec.}

treffen auch für das mittlere Lahntal zu. In der Nadelwaldphase im Aschafftal bei Hösbach ist ebenfalls die Tanne am Waldaufbau beteiligt, allerdings mit weitaus geringeren Werten, als es in den Pollenanalysen der Mosbacher Humuszonen anklingt. Neben Fichte, Kiefer und Lärche war

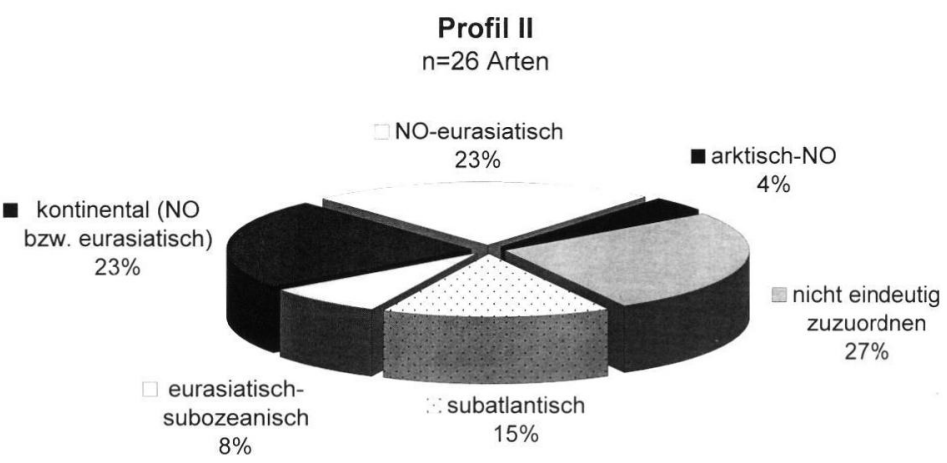

Abb. 9: Geographische Verbreitungsschwerpunkte der wichtigsten botanischen Großreste aus Profil Niederweimar II (Angaben nach OBERDORfER

Fig. 9: Main geographical distributions of important botanical macro remains from the profile Niederweimar II (Details from OBERDORFER 1990). 
auch die Pappel im Auewald bei Hösbach vertreten. Ein Vegetationsbild, das dem der mittleren Lahn während des Brörup-Interstadials entspricht. Die Elemente des Eichenmischwaldes sind im Pollendiagramm von Hösbach subsummiert, so dass sich keine detaillierten Aussagen über die Anwesenheit von Eiche und Ulme machen lassen. Als weitere Punkte werden noch aufgeführt:

- Auftreten von Betula nana und Betula bumilis

- Massenentwicklung von Carex aquatilis.

Makrorestfunde von Betula bumilis aus dem Brörup-Interstadial (BEHRE 1974, LiTT 1994, FREUND 1997) sowie neue Untersuchungen durch FREUND et al. (in Vorbereitung), belegen eindeutig, dass die Strauch-Birke im nördlichen Mitteleuropa sowohl in den Interstadialen als auch in Stadialen des Weichselfrüh- und Hochglazials vertreten war. Ihre Wuchsorte lagen vorzugsweise in Bruchwäldern und auf Flachmooren. Ein Auftreten zusammen mit der Zwerg-Birke ist vor allem für den Beginn und das Ende des Interstadiales nicht außergewöhnlich, wobei Betula nana im Weichsel-Frühglazial aber vermutlich auch auf Mineralböden wuchs. Die Untersuchungsergebnisse von BaAs (1986) würden sich hier nahtlos einreihen. Gleiches gilt für die Funde der Wasser-Segge (Carex aquatilis), die, teilweise in großer Zahl, durch BeHRe (1974) und Freund (1997) ebenfalls im Brörup-Interstadial nachgewiesen wurde.

Die pollen- und makrorestanalytischen Untersuchungen im mittleren Lahntal dienten zum einen der chronostratigraphischen Einordnung des unteren Schotterkomplexes. Zum anderen fanden aber auch paläoklimatische Aspekte eine besondere Berücksichtigung; denn bei der Beurteilung und Abschätzung von Ausbreitungsrichtung, -geschwindigkeit und -grenzen bestimmter Arten, sowie zur Quantifizierung des nord-süd-gerichteten Klimagradienten im Früh-Weichselglazial kommt den Profilen aus dem mittleren Lahntal eine besondere Bedeutung zu.

Betrachtet man die in Abb. 9 dargestellten geographischen Verbreitungsschwerpunkte, fällt neben den heute eher nordisch und kontinental verbreiteten Arten besonders der hohe Anteil eurasiatisch-subozeanischer und subatlantischer Arten ins Auge. Diese Verteilung weist darauf hin, dass das frühweichselzeitliche Klima im hessischen Mittelgebirgsraum nicht extrem kontinental getönt war. Daher bietet sich ein Vergleich mit den von LitT (1994) und von CASPERs \& Freund
(1997) vorgelegten Klimarekonstruktionen für das Brörup-Interstadial an.

Vor allem das gemeinsame Auftreten von Larix spec., Picea abies, Pinus sylvestris und verschiedenen Betula-Arten (B.pubescens, B. alba, B. bumilis, B. nana) führte bislang zu der Auffassung, dass im Brörup-Interstadial ein extrem kontinentales, winterkaltes Klima geherrscht haben musste (BEHRE 1974). Dagegen spricht das zeitgleiche Auftreten vieler subozeanisch verbreiteter Sippen (Menke \& Tynn 1984, LitT 1994). Wie oben aufgeführt, fügen sich in Niederweimar vor allem die Nachweise von Sambucus nigra (M= Makrorest), Luronium natans (M), Peplis portula (M), Pilularia globulifera $(\mathrm{S}=\mathrm{Spore})$ und Corrigiola litoralis (M) in dieses Bild (ausgewählte Makroreste zeigt Taf. 1).

In der Hösbacher Flora von BAAs (1986) kommen mit Isoëtes echinospora (S), Osmunda regalis (S), Clematis vitalba (M) und Nymphaea alba (M) weitere Arten hinzu, der Nachweis von Sambucus nigra (M) deckt sich mit dem Vorkommen im Lahntal. In jüngster Zeit hat sich auch die Untersuchung fossiler Käferreste als überaus hilfreiches Werkzeug zur Rekonstruktion sowohl des Paläoklimas als auch der Landschaftsgenese erwiesen. Käfer-Analysen von WALKLING (1997) an brörupzeitlichen Ablagerungen der Bohrung Groß Todtshorn ergaben sehr milde mittlere Wintertemperaturen von ca. $-3{ }^{\circ} \mathrm{C}$ im kältesten Monat.

Dabei ist zu berücksichtigen, dass Wintertemperaturen generell schlechter zu quantifizieren sind als die Sommertemperaturen. Eine mittlere Temperatur des kältesten Monats um $-10^{\circ} \mathrm{C}$ erschein daher für Nordwestdeutschland realistischer. Die von LINDROTH (in BAAs 1986: 350 ff) überprüften entymologischen Bestimmungen von FLACH (1884) ergaben eine Käfervergesellschaftung, die sich mit dem skandinavischen Nadelwaldgebiet oder der russischen Taiga vergleichen ließ, was ebenfalls auf durchschnittliche Temperaturen im kältesten Monat von ca. $-15^{\circ} \mathrm{C}$ hindeutet. Die von LiтT (1994) vorgeschlagene Boreorussische Provinz als Modellregion für das brörupzeitliche Klima ist somit auch auf den Bereich der südlichen Mittelgebirgsschwelle übertragbar. Vermutlich lag die Niederschlagsmenge aber höher als in den Gebieten Mittel- und Ostdeutschlands, was, aufgrund der pollenanalytischen Ergebnisse, die Vergleichbarkeit zu den für Nordwestdeutschland erarbeiteten Diagrammen erklärt (CASPERS \& FrEUND 1997). Die Temperaturen im wärmsten Monat lagen im Mittel über $14^{\circ} \mathrm{C}$, wobei höhere 
Temperaturen in Süddeutschland wahrscheinlich sind. Die Wintertemperaturen waren im Mittel des kältesten Monats überall deutlich wärmer als $-20{ }^{\circ} \mathrm{C}$, wobei natürlich auch hier lokalklimatische Modifikationen auftraten.

Die Gründe, warum anspruchsvollere Bäume wie Eiche oder Ulme, die in süddeutschen und nordfranzösischen Pollendiagrammen verzeichnet sind, nicht weiter nach Norden vorgestoßen sind, müssen daher woanders liegen. Vermutlich waren es die Niederschlagsverhältnisse und die Länge der Vegetationsperiode, die besonderen Einfluss auf die Vegetationszusammensetzung hatten. So verhinderten eine lange winterliche Schneebedeckung im Westen sowie lange, und vor allem trockene Winter im Osten die Ausbreitung anspruchsvollerer Laubbäume im mitteleuropäischen Flachland. Wärmeliebende Laubgehölze, wie zum Beispiel Eiche und Ulme, breiteten sich demnach nur bis in die Tieflagen der Mittelgebirge aus, wo sie ihre nördliche Verbreitungsgrenze fanden.

Wie die Untersuchungen an der mittleren Lahn, aber auch im Aschafftal bei Hösbach zeigen, bieten vor allem organogene Einschaltungen in Terrassen (Paläo-Rinnen etc.) eine gute Möglichkeit, weitere weichselzeitliche Interstadiale im bislang fundleeren Raum zwischen Nord- und Süddeutschland zu finden.

\section{Dank}

Für die Anregung zur Untersuchung der LahnNiederterrasse sind wir Herrn Prof. Dr. R. HuckRIEDE (Marburg) zu besonderem Dank verpflichtet. Frau Dipl.-Geogr. A. Schweizer (Frankfurt/Main) sei ganz herzlich für die Erstellung der Großrestdiagramme gedankt. Die Arbeiten am REM wurden von Herrn Dr. A. SCHAPER (Marburg) durchgeführt, auch ihm sei an dieser Stelle herzlich gedankt. Den Mitarbeitern der Firma LahnWaschkies, Readymix Kies Saar-Pfalz-Hessen $\mathrm{GmbH}$ in Heuchelheim und Weimar-Niederweimar danken wir für die Unterstützung bei den Geländearbeiten. Weiterhin gilt unser Dank den Herren Prof. Dr. K.-E. Behre (Wilhelmshaven) und Prof. Dr. T. LiTT (Bonn) für Anregungen und die kritische Durchsicht des Manuskripts.

\section{Schriftenverzeichnis}

BAAs, J. (1986): Die Vegetationsgeschichte der mittelpleistozänen Tone von Hösbach bei Aschaffenburg am Main. - Cour. Forsch.-Inst. Senckenberg, 86: 331-385, 10 Abb., 5 Taf.; Frankfurt/M.
BeHre, K.-E. (1974): Die Vegetation im Spätpleistozän von Osterwanna/Niedersachsen. - Geol. Jb., A 18: 3-48, 8 Abb., 5 Taf.; Hannover.

Bibus, E., Bludau, W., Bross, C. \& Rähle, W. (1996): Der Altwürm- und Rißabschnitt im Profil Mainz-Weisenau und die Eigenschaften der Mosbacher Humuszonen. - In: Semmel, A. [Hrsg.]: Pleistozäne und holozäne Böden aus Lößsubstraten am Nordrand der oberrheinischen Tiefebene. - Frankfurter geowiss. Arb., Serie D, 20: 21-52; Frankfurt/M.

Caspers, G. (1997): Die eem- und weichselzeitliche Hohlform von Groß Todtshorn (Kr. Harburg; Niedersachsen) - Geologische und palynologische Untersuchungen zu Vegetation und Klimaverlauf der letzten Kaltzeit. - Schriftenreihe dt. geol. Ges., 4: 759, 39 Abb., 6 Tab.; Hannover.

- \& Freund, H. (1997): Die Vegetations- und Klimaentwicklung des Weichsel-Früh- und -Hochglazials im nördlichen Mitteleuropa. - Schriftenreihe dt. geol. Ges., 4: 201-249, 31 Abb., 4 Tab.; Hannover.

- \& FREUND, H. (2000/in Vorbereitung): Vegetation and climate in the Early- and Pleni-Weichselian in northern central Europe. - J. Quat. Science.

ERD, K. (1973): Pollenanalytische Gliederung des Pleistozäns der Deutschen Demokratischen Republik. Z. geol. Wiss., 1: 1087-1103, 7 Abb., 2 Tab.; Berlin.

Flach, K. (1884): Die Käfer der unterpleistozänen Ablagerungen bei Hösbach unweit Aschaffenburg. Verh. phys. med. Ges., N.F. 18: 285-297, 2. Taf.; Würzburg.

FreuND, H. (1997): Die Vegetation im Weichsel-Frühund -Hochglazial von Groß Todtshorn (Kr. Harburg; Niedersachsen) anhand botanischer Makroreste. - Schriftenreihe dt. geol. Ges., 4: 61-86, 12 Abb., 2 Tab., 5 Fotos; Hannover.

- Birks, H. H. \& Birks, H. J. B. (in Vorbereitung): The identification of wingless Betula fruits in Weichselian sediments in the Gross Todtshorn borehole (Lower Saxony, Germany); the occurence of Betula humilis. - Veg. Hist. and Archaeobotany.

Grimm, E. C. (1992): TILIA and TILIA-graph: pollen spreadsheet and graphics programs. - Volume of abstracts 8th International Palynological Congress 1992: 56; Aix-en-Provence.

GrüGER, E. (1979): Die Seeablagerungen vom Samerberg/Obb. und ihre Stellung im Jungpleistozän. Eiszeitalter u. Gegenwart, 29: 23-34, 4 Abb., 1 Tab.; Hannover.

Heine, K. (1970): Fluß- und Talgeschichte im Raum Marburg. Eine geomorphologische Studie. - Bonner geogr. Abh., 42: 195 S., 49 Abb.; Bonn.

Hölting, B. \& Stengel-Rutkowski, W. (1964): Beiträge zur Tektonik des nordwestlichen Vorlandes des basaltischen Vogelsberges, insbesondere des Amöneburger Beckens. - Abh. hess. L.-Amt Bodenforsch., 47: 1-37; Wiesbaden.

Huckriede, R. (1972): Der Untergrund des Deutschen Hauses und weitere geologische und urgeschichtliche Befunde in Marburg an der Lahn. - Geologica et Palaeontologica, 6: 177-201; Marburg/Lahn.

- (1982): Paläoklimatische Aussagen neuer weichselzeitlicher Pflanzenfunde in Hessen und Tirol. - Physische Geographie, 5: 37-38; Zürich. 
LiTr, T. (1994): Paläoökologie, Paläobotanik und Stratigraphie des Jungquartärs im nordmitteleuropäischen Tiefland - Unter besonderer Berücksichtigung des Elbe-Saale-Gebietes. - Diss. Bot., 227: 185 S., 50 Abb., 10 Tab., 4 Beil.; Berlin, Stuttgart.

Menke, B. \& Tynni, R. (1984): Das Eeminterglazial und das Weichsel-Frühglazial von Rederstall/Dithmarschen und ihre Bedeutung für die mitteleuropäische Jungpleistozän-Gliederung. - Geol. Jb., A 76: 120 S., 18 Abb., 7 Tab., 9 Taf.; Hannover.

OBERdorfer, E. (1990): Pflanzensoziologische Exkursionsflora. - 6. Aufl.: 1050 S., 58 Abb.; Stuttgart (Ulmer).

- (1992): Süddeutsche Pflanzengesellschaften. Teil IV: Wälder und Gebüsche - 2. Aufl.: 282 S., 3 Abb., 104 Tab; Stuttgart (Fischer).
Ротт, R. (1995): Die Pflanzengesellschaften Deutschlands. - 2. Aufl.: 622 S., 449 Abb.; Stuttgart (Ulmer).

Urz, R. (1995): Jung-Quartär im Auenbereich der mittleren Lahn - Stratigraphische und paläontologische Untersuchungen zur Rekonstruktion vergangener Flußlandschaften. - Diss. Fachber. Geowiss., Univ. Marburg/Lahn: 198 S., 28 Abb., 3 Tab., 5 Taf,; Marburg/Lahn.

WaLKLING, A. (1997): Käferkundliche Untersuchungen an weichselzeitlichen Ablagerungen der Bohrung Groß Todtshorn (Kr. Harburg; Niedersachsen). Schriftenreihe dt. geol. Ges., 4: 87-102, 17 Abb.; Hannover.

Manuskript eingegangen am 20. 12. 1999, zum Druck angenommen am 20. 06. 2000 